\title{
Improved Eddy-current Field Loss Model and Scaling Index for Magnets of Permanent Magnet Synchronous Motors
}

\author{
Lei Zhang $^{1, a}$, Chunxia Gao ${ }^{1}$ \\ ${ }^{1}$ College of Information and Control Engineering, China University of Petroleum, Qingdao, China
}

\begin{abstract}
The paper gives detailed systematic researches on the mechanism and key factors of eddy-current losses in rotor magnets of high power-density permanent magnet synchronous motors(PMSMs).Firstly, this paper establishes quantitative mathematic model of eddy-current losses for surface-mounted PMSM based on eddy current field model and Maxwell equations. Then, a scaling index is put forward to weigh the key factors relevant to the eddy-current losses in magnets. At the same time, the principles of eddy-current losses in prototype PMSM are analyzed by the finite element analysis (FEA) software. The contents researched in the paper have practical reference values for design and reliability analysis of PMSMs.
\end{abstract}

\section{Introduction}

Nowadays, permanent magnet synchronous motors (i.e. PMSMs) have become one researching hotspot in the electric vehicle and other high power-density fields, for the advantages of high power density, simple structure, high energy-efficiency, and of long-life operating cycles but low maintaining costs. For the purpose of higher power-density, it is very difficult to make major breakthrough in mechanical materials but to increase the revolving speed of the motors[1].

Literature [2-5] investigate the eddy current loss based on the finite element analysis, especially the influence of segmentation in magnets. But the analyses lack the support of theories and relative analyses are carried out based on the uniformly alternating magnetic field which do not consider the asymmetric distribution and influencing weight of different harmonics.

In this paper, an improved quantitative mathematic model of eddy-current losses for surface-mounted PMSM, based on eddy current field model and Maxwell equations, is established. Then, a scaling index is put forward to weigh the key factors relevant to the eddy-current losses in magnets. At the same time, the principles of eddycurrent losses in prototype PMSM are analyzed with the finite element analysis software.

\section{Improved Mathematic Model of Eddy- Current in Magnets}

\subsection{Still Model Based on Eddy-Current Field Model}

It is assumed that:

a techupc@gmail.com
1. Motor has inner rotor structure with uniformly magnetized magnets and nonconducting retaining sleeve. The inner diameter of stator and outer diameter of rotor are large enough to be approximately expended along the air-gap.

2. The end effect of eddy-current is neglected and the distribution of eddy-current along the $\mathrm{z}$-axis is unchanged. 3. Neglecting the saturation effect of rotor core and slotting effect of stator in analytical study.

4. Stator currents can be equivalent to the circumferential current density sheet and $\mathrm{k}$ is its unit vector along the $\mathrm{z}$ axis.

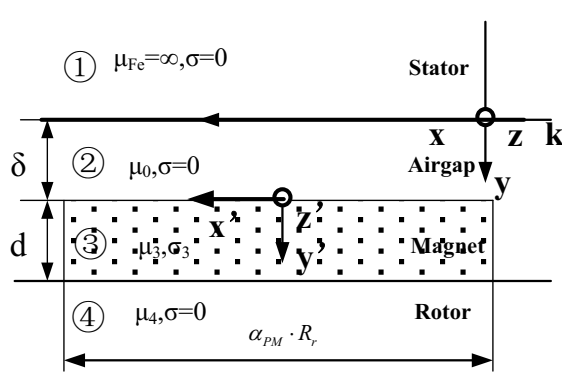

Figure 1. Equivalent coordinate system in the air-gap

When ignoring the time harmonic components and imposing the symmetric 3-phase sine-wave current, the equivalent MMF is:

$$
\begin{aligned}
& F(x, t)=N_{A} i_{\mathrm{A}}+N_{\mathrm{B}} i_{\mathrm{B}}+N_{\mathrm{C}} i_{\mathrm{C}} \\
& =\left.\sum_{n \in R}^{\infty} N_{c n} I_{m} \kappa_{ \pm_{1} n} \cos \left(\omega \mathrm{f}+\alpha_{0 n} \pm_{1} n \frac{\pi}{\tau} x\right)\right|_{\kappa_{n}=\left(\frac{1}{2}+\cos \left((n+1) \frac{2 \pi}{3}\right)\right)} \\
& =\sum_{n \in R}^{\infty} f_{\mathrm{cn}} \cos \left(\omega \mathrm{f}+\alpha_{0 n} \text { 犃 } n \frac{\pi}{\tau} x\right)=\sum_{n \in R}^{\infty} f_{\mathrm{cr}} \cos \left({ }_{\mathrm{r}} \mathrm{t}+\alpha_{0 n}+v \frac{\pi}{\tau} x\right)
\end{aligned}
$$




\subsection{Eddy-Current Model in Rotor Coordinate}

\subsubsection{Analytical Equations of Flux Density and Eddy- Current Density}

According to the eddy-current field model, the influence of armature reaction is relative to the rotor coordinate. As a result, the analytical equations should be transformed to the rotational rotor coordinate system. So, the eddycurrent density in magnets can be described as:

$$
\begin{aligned}
& J_{z 3 v}(x, t)= \\
& \operatorname{Re}\left[\frac{\mu_{0} \cdot J_{v} \cdot(1+v) \omega_{r} \sigma \operatorname{ch} \beta(d+\delta-y)}{\beta \cdot \operatorname{ch} \frac{\pi}{\tau} v \delta \cdot \operatorname{sh} \beta d+\frac{\pi}{\tau} v \cdot \operatorname{sh} \frac{\pi v \delta}{\tau} \operatorname{ch} \beta d} e^{j\left((1+v) \omega \mathrm{f}+\alpha_{0 v}+v \frac{\pi}{\tau} x\right)}\right]
\end{aligned}
$$

\subsubsection{Analytical Formula for the Eddy-Current Loss}

Making use of loss formula, the eddy-current loss equation can be shown as:

$$
P=\frac{1}{2} \int_{V} \operatorname{Re}\left\{\dot{\boldsymbol{E}} \times \dot{\boldsymbol{J}}^{*}\right\} \cdot d V
$$

Thus, we can define a constant $\kappa$ as the index for the magnets in order to research the influential factor of eddy-current loss, which provide the way to scale the eddy-current loss but without the calculation of double integral:

$$
\kappa=\log \left(\sum_{v \in R}\left[\mu_{0} \cdot N_{v} \cdot(1+v) \cdot \omega_{r} \cdot \zeta\right]^{2} \cdot \sigma \cdot l_{e f f} \cdot d \cdot \alpha_{P M} R_{r}\right)
$$

And $\mathrm{Wi}$ is the weight factor which can revise the factor $\kappa$ according the different structure.

Making use of equation 4, Table 1 can be obtained to

\begin{tabular}{|c|c|c|c|c|c|c|c|c|c|c|c|c|c|c|}
\hline $\mathbf{Q} / \mathbf{2 p}$ & 4 & 6 & 8 & 10 & 12 & 14 & 16 & 18 & 20 & 22 & 24 & 26 & 28 & 30 \\
\hline 6 & -4.2 & & -1.22 & -0.83 & & -1.23 & -1.45 & & -1.01 & -1.20 & & -1.42 & -1.31 & \\
\hline 9 & -1.3 & -4.31 & -2.24 & -2.20 & -1.85 & $\begin{array}{l}-0.96 \\
\end{array}$ & -1.05 & & -1.30 & -1.26 & -1.88 & -2.12 & -2.09 & -1.39 \\
\hline 12 & 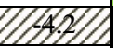 & & -3.90 & -1.97 & & -2.36 & -2.66 & & --- & --- & & --- & --- & \\
\hline 15 & & & -2.56 & -3.69 & & -2.56 & -2.63 & & -3.88 & -2.21 & & -- & --- & \\
\hline 18 & & 14021 & -1.85 & -2.28 & -3.57 & -2.46 & $\begin{array}{l}-2.62 \\
\end{array}$ & & -2.24 & -2.31 & & -2.82 & --- & --- \\
\hline 21 & & & -1.86 & --- & & -3.50 & -2.50 & & -2.63 & -2.59 & -2.91 & -2.09 & -2.47 & \\
\hline 24 & & & $1-3.94$ & --- & & -2.74 & -3.44 & & -2.00 & -2.61 & & -2.48 & -2.10 & \\
\hline 27 & & & & --- & --- & --- & --- & -3.41 & -2.69 & -2.41 & -2.72 & -2.60 & -2.51 & -2.27 \\
\hline & & \multicolumn{6}{|c|}{ Wingding factor $=0.866$} & \multicolumn{6}{|c|}{ Not exist } & \\
\hline & --- & \multicolumn{5}{|c|}{ Small wingding factor } & & \multicolumn{6}{|c|}{ Winding factor $=1$} & \\
\hline
\end{tabular}
research the principle of eddy-current losses of different slot-pole combinations.

Table 1 Scaling index of different slot-pole combinations

\section{Comparisons between FEA and Analytical Simulation}

In order to verify the validation of the analytical formulae, the comparisons between FEA and analytical simulations are given in the following under the same conditions.

\subsection{Flux Density in the Air-Gap}

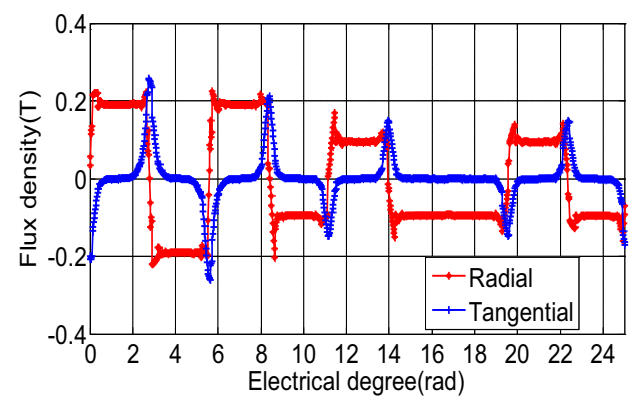

(a) FEA

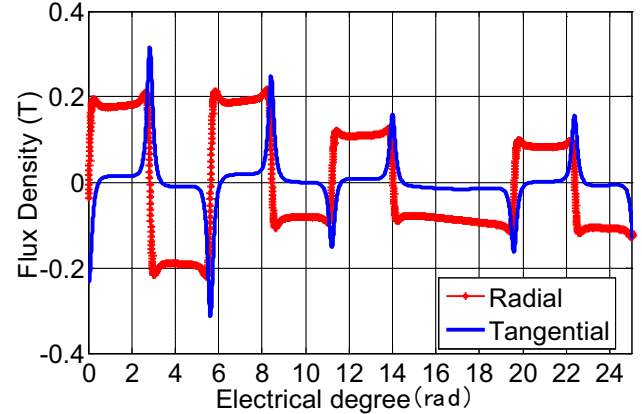

(b) Analytical

Figure 2. Radial and tangential flux density along circumference

From the comparisons between FEA and analytical model, it is seen that the simulation results meet well with each other, which prove the validity of analytical model.

It is seen that the flux density in magnet is not constant but varying with time and meets well with each other. 


\subsection{Eddy-Current Density of One Point in Magnets}

Because of high conductivity of the magnet, very small change in flux density can cause large eddy-current density, which is one of the reasons for the high eddycurrent loss in high power-density motors. It is seen from Fig.3 that analytical eddy-current density meets fairly well with FEA results from low to high frequency, which can ensure the accuracy of estimation of eddy-current loss.

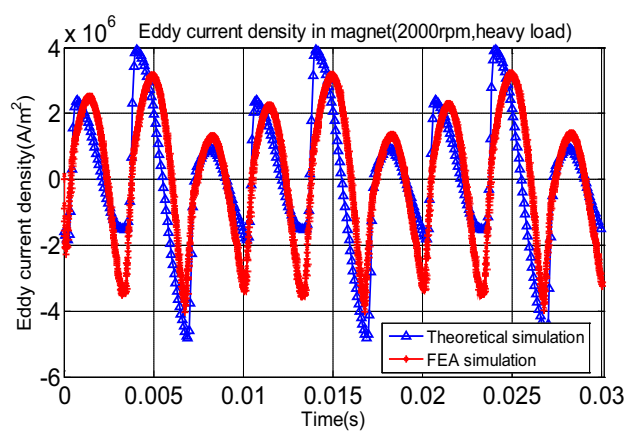

(a) Eddy current density in magnets at 2000rpm

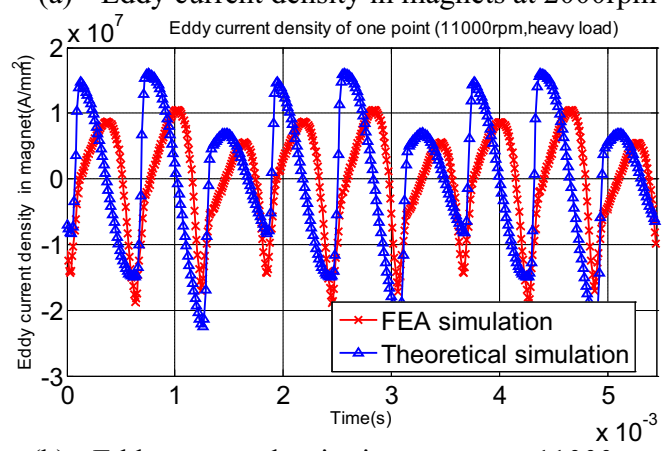

(b) Eddy current density in magnets at $11000 \mathrm{rpm}$

Figure 3. Eddy-current density of one point in magnet in analytical simulation

\subsection{Scaling Index $\kappa$}

In order to validate the effectiveness of index $\kappa$, comparative researches are carried out for four types of prototype motors based on Equation(4): while 8-pole/9slot is a typical concentrated-winding fractional-slot (CWFS) PMSM; 8-pole/12-slot is a distributed-winding fractional-slot (DWFS) PMSM; 8-pole/24-slot is a $\mathrm{q}=1$ (q is slots per-pole per-phase) integral-slot PMSM; and 8pole/48-slot is a typical distributed-winding integral-slot (DWIS) PMSM.

Although these four type of motors have the same stator current, the harmonic component in flux density are very different for the space harmonics: CWFS structures contain much more harmonics, especially subharmonic components which can produce large losses in magnets, than other type of structure.

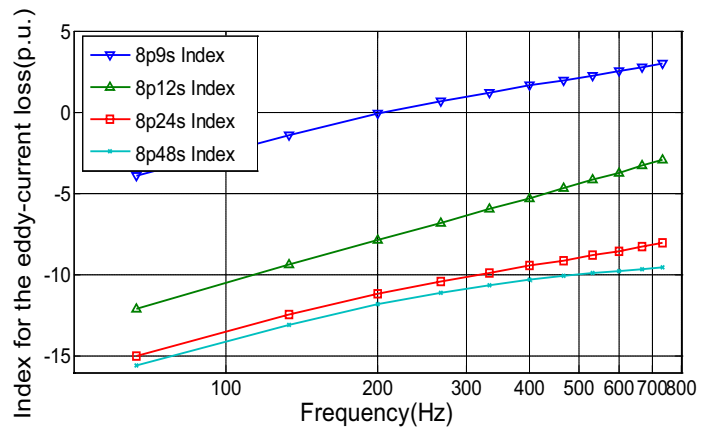

(a) Eddy-current loss index for prototype motors

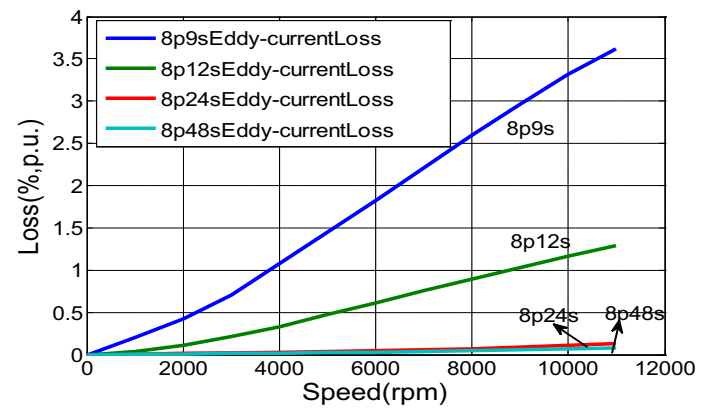

(b) Eddy-current loss for prototype motors

Figure 4. Scaling index and eddy-current loss in prototype motors

It is shown in Fig. 4 that the index can directly make out the inherent characteristics of eddy-current-loss in magnets for prototype motors: as for the CWFS PMSM (e.g. 8-pole/9-slot) contains more remarkable harmonics in magnetic field and eddy-current loss than other motors; comparatively, distributed-winding fractional-slot PMSMs (e.g. 8-pole/12-slot) are better than CWFS type of motors. And the distributed-winding integral-slot type motors are much better than the above-mentioned types of motors.

\section{Summary}

Generally speaking, rotor losses in PMSMs are commonly neglected in tradition loss analysis, whereas the engineering practices reveal that some type of structure (especially fractional-slot) motors do exist the risk of over-heating demagnetization problem for the inner eddy-current losses of magnets when operating in large power, high speed conditions.

In order to deeply analyze the eddy-current loss in magnets, research of mechanism and influential factors are performed in detailed in this paper. An improved loss model for surface PMSM is deduced to simplify the analysis of existing theory. Also a scaling index $\mathrm{k}$ is put forward to weight the influential factors of eddy-current loss in magnets. Meanwhile, comparative researches between analytical and finite element analysis are performed, which show the good agreement for these two models. 


\section{References}

1. H. Surong, L. Jian, F. Leonardi, and T. A. Lipo, " $A$ general approach to sizing and power density equations for comparison of electrical machines," IEEE Transactions on Industry Applications, vol. 34, pp. 92-97, 1998.

2. K. Yamazaki and Y. Fukushima, "Effect of eddycurrent loss reduction by magnet segmentation in synchronous motors with concentrated windings," in Electrical Machines and Systems, 2009. ICEMS 2009. International Conference on, (2009), pp. 1-6.

3. C. Deak, L. Petrovic, A. Binder, M. Mirzaei, D. Irimie, and B. Funieru, "Calculation of eddy current losses in permanent magnets of synchronous machines," in International Symposium on Power Electronics, Electrical Drives, Automation and Motion, 2008. SPEEDAM 2008., (2008), pp. 26-31.

4. K. Yamazaki, S. Ohki, A. Nezu, and T. Ikemi, "Development of Interior Permanent Magnet Motors Reduction of Harmonic Iron Losses by Optimizing Rotor Structures," in IEEE International Electric Machines \& Drives Conference, 2007. IEMDC '07., (2007), pp. 489-494.

5. K. Yamazaki, "Rotor-Shape Optimization of InteriorPermanent-Magnet Motors to Reduce Harmonic Iron Losses," IEEE Transactions on Industrial Electronics, vol. 57, pp. 61-69, 2010. 